\title{
Diameter, extreme points and topology
}

by

\section{J. C. Navarro-Pascual and M. G. Sanchez-Lirola (Almería)}

Abstract. We study the extremal structure of Banach spaces of continuous functions with the diameter norm.

1. Introduction. Let $X$ be a Banach space. The closed unit ball of $X$ and the set (maybe empty) of its extreme points will be denoted by $B_{X}$ and $E_{X}$, respectively. As usual, we denote by $\operatorname{co}\left(E_{X}\right)$ the convex hull of $E_{X}$ and by $\overline{c o}\left(E_{X}\right)$ its closure, that is, the closed convex hull of $E_{X}$.

From now on, $K$ will stand for a compact Hausdorff space and $C(K)$ will be the space of continuous functions from $K$ into $\mathbb{R}$. For every $f \in C(K)$, $\varrho(f)$ will denote the diameter of $f(K)$ :

$$
\varrho(f)=\max \left\{\left|f(t)-f\left(t^{\prime}\right)\right|: t, t^{\prime} \in K\right\} .
$$

It is clear that $\varrho$ is a seminorm on $C(K)$ and, given $f \in C(K), \varrho(f)=0$ if, and only if, $f$ is a constant function. The quotient of $C(K)$ by the constant functions becomes a Banach space with respect to the diameter by defining $\varrho([f])=\varrho(f)$ for every $f \in C(K)$.

Alternatively, we can fix a point $t_{0}$ in $K$ and consider the following subspace of $C(K)$ :

$$
X=\left\{f \in C(K): f\left(t_{0}\right)=0\right\} .
$$

Below we will work with this subspace, which is - for the diameter norm-a Banach space isometric to the above-mentioned quotient. In order to avoid the trivial case we will suppose that $K$ has at least two points. The diameter norm on $X$ is equivalent to the uniform norm. In fact,

$$
\|f\|_{\infty} \leq \varrho(f) \leq 2\|f\|_{\infty} \quad \text { for every } f \in X .
$$

In recent years, several authors have been considering the diameter to get Banach-Stone type theorems. In this connection, we mention the first work on diameter preserving linear bijections by M. Győry and L. Molnár [2]. 
However, our purpose is different. We will obtain Krein-Milman type theorems in spaces of continuous functions with the diameter norm.

2. The results. We start with a useful description of extreme points of the unit ball of $X$.

Lemma 1. The extreme points of $B_{X}$ are the functions $e \in X$ such that $e(K)=\{0,1\}$ or $e(K)=\{-1,0\}$.

Proof. Let $e$ be in $X$ such that $e(K)=\{0,1\}$ or $e(K)=\{-1,0\}$ and take $f, g \in X$ with $\varrho(f) \leq 1, \varrho(g) \leq 1$ and $e=(f+g) / 2$. Given $t \in K$ with $|e(t)|=1$, it is obvious that $e(t)=f(t)=g(t)$. Suppose that $e(t)=0$ and fix a point $t^{\prime} \in K$ such that $\left|e\left(t^{\prime}\right)\right|=1$. Then $e\left(t^{\prime}\right)=f\left(t^{\prime}\right)=g\left(t^{\prime}\right)$ and $e\left(t^{\prime}\right)=e\left(t^{\prime}\right)-e(t)=\frac{f\left(t^{\prime}\right)+g\left(t^{\prime}\right)}{2}-\frac{f(t)+g(t)}{2}=\frac{f\left(t^{\prime}\right)-f(t)}{2}+\frac{g\left(t^{\prime}\right)-g(t)}{2}$.

Taking into account that $\left|e\left(t^{\prime}\right)\right|=1$ and $\left|f\left(t^{\prime}\right)-f(t)\right| \leq 1,\left|g\left(t^{\prime}\right)-g(t)\right| \leq 1$, it follows that

$$
e\left(t^{\prime}\right)=f\left(t^{\prime}\right)-f(t)=g\left(t^{\prime}\right)-g(t),
$$

and consequently $e(t)=0=f(t)=g(t)$.

Conversely, let $e$ be an extreme point of the unit ball of $X$. For each $t$ in $K$ define

$$
\alpha(t)=\max \left\{\left|e(t)-e\left(t^{\prime}\right)\right|: t^{\prime} \in K\right\}
$$

and choose a point $t_{1} \in K$. Suppose, to obtain a contradiction, that $\alpha\left(t_{1}\right)<1$ and let $\varepsilon$ be in ]0, $\left(1-\alpha\left(t_{1}\right)\right) / 2$ [. Let us consider a continuous function $\varphi$ : $K \rightarrow[0,1]$ such that

$$
\varphi(t)= \begin{cases}1 & \text { if }\left|e(t)-e\left(t_{1}\right)\right| \leq \varepsilon, \\ 0 & \text { if }\left|e(t)-e\left(t_{1}\right)\right| \geq 1-\alpha\left(t_{1}\right) / 2,\end{cases}
$$

and let $t, t^{\prime}$ be in $K$. First suppose that $\left|e(t)-e\left(t_{1}\right)\right| \leq\left(1-\alpha\left(t_{1}\right)\right) / 2$. Then

$$
\begin{aligned}
& \left|\left(e(t) \pm \frac{1-\alpha\left(t_{1}\right)}{2} \varphi(t)\right)-\left(e\left(t^{\prime}\right) \pm \frac{1-\alpha\left(t_{1}\right)}{2} \varphi\left(t^{\prime}\right)\right)\right| \\
& \quad=\left|e(t)-e\left(t^{\prime}\right) \pm \frac{1-\alpha\left(t_{1}\right)}{2}\left(\varphi(t)-\varphi\left(t^{\prime}\right)\right)\right| \\
& \quad \leq\left|e(t)-e\left(t^{\prime}\right)\right|+\frac{1-\alpha\left(t_{1}\right)}{2} \leq\left|e(t)-e\left(t_{1}\right)\right|+\left|e\left(t_{1}\right)-e\left(t^{\prime}\right)\right|+\frac{1-\alpha\left(t_{1}\right)}{2} \\
& \quad \leq \frac{1-\alpha\left(t_{1}\right)}{2}+\alpha\left(t_{1}\right)+\frac{1-\alpha\left(t_{1}\right)}{2}=1 .
\end{aligned}
$$

Of course, the same holds if $\left|e\left(t^{\prime}\right)-e\left(t_{1}\right)\right| \leq\left(1-\alpha\left(t_{1}\right)\right) / 2$. On the other hand, if $\left|e(t)-e\left(t_{1}\right)\right| \geq\left(1-\alpha\left(t_{1}\right)\right) / 2$ and $\left|e\left(t^{\prime}\right)-e\left(t_{1}\right)\right| \geq\left(1-\alpha\left(t_{1}\right)\right) / 2$, we 
have

$$
\left|\left(e(t) \pm \frac{1-\alpha\left(t_{1}\right)}{2} \varphi(t)\right)-\left(e\left(t^{\prime}\right) \pm \frac{1-\alpha\left(t_{1}\right)}{2} \varphi\left(t^{\prime}\right)\right)\right|=\left|e(t)-e\left(t^{\prime}\right)\right| \leq 1
$$

This proves that $\varrho\left(e \pm \frac{1-\alpha\left(t_{1}\right)}{2} \varphi\right) \leq 1$ and contradicts, since $\varphi \neq 0$, the fact that $e$ is an extreme point of the unit ball of $X$.

Consequently, since $t_{1}$ was arbitrary in $K, \alpha(t)=1$ for every $t \in K$. In particular, $\alpha\left(t_{0}\right)=1$, and thus $e(K) \cap\{-1,1\} \neq \emptyset$. If $1 \in e(K)$ it is clear that $0 \leq e(t) \leq 1$ for each $t \in K$ and, according to the above, there is not a point $t$ in $K$ such that $0<e(t)<1$. Therefore, $e(K)=\{0,1\}$. A similar argument proves that $e(K)=\{-1,0\}$ if $-1 \in e(K)$.

As an immediate consequence, we have

Corollary 2. $E_{X} \neq \emptyset$ if, and only if, $K$ is not connected.

It is worth noting that, in general, the geometry of $X$ with the diameter norm is different from its geometry with the uniform norm. For instance, if $K$ does not have isolated points and it is not connected, then $E_{X}$ is nonempty by the previous lemma but the unit ball of $X$ with the uniform norm does not contain extreme points.

On the other hand, the preceding corollary emphasizes that the extremal structure of $X$ depends on the degree of disconnectedness of $K$.

Let us recall that a topological space is totally disconnected if every element has a neighbourhood base of closed and open sets.

THEOREM 3. The following assertions are equivalent:

(i) For each $x \in B_{X}$ there is a sequence $\left\{\lambda_{n}\right\}$ in $[0,1]$ with $\sum_{n=1}^{\infty} \lambda_{n}=1$ and a sequence $\left\{e_{n}\right\}$ of extreme points of the unit ball of $X$ such that $x=\sum_{n=1}^{\infty} \lambda_{n} e_{n}$.

(ii) $B_{X}=\overline{\mathrm{co}}\left(E_{X}\right)$.

(iii) $K$ is totally disconnected.

Proof. It is clear that (i) implies (ii).

In order to prove that (ii) implies (iii), let $t_{1}$ be an element of $K$ with $t_{1} \neq t_{0}$. Let $U$ and $V$ be open and disjoint neighbourhoods of $t_{0}$ and $t_{1}$, respectively. Select two continuous functions $x, y: K \rightarrow[0,1]$ such that $x\left(t_{0}\right)=0$ and $x(t)=1$ for every $t \in K \backslash U$, and $y\left(t_{1}\right)=1$ and $y(t)=0$ for each $t \in K \backslash V$. Obviously $x, y \in B_{X}$ and, by (ii), there are $f, g \in \operatorname{co}\left(E_{X}\right)$ such that $\varrho(f-x)<1 / 2$ and $\varrho(g-y)<1 / 2$. Taking into account that $f(K)$ and $g(K)$ are finite, the sets $U_{1}=\{t \in K: f(t) \leq 1 / 2\}$ and $V_{1}=\{t \in K: g(t) \geq 1 / 2\}$ are open and closed. Furthermore, $t_{0} \in U_{1} \subset U$ and $t_{1} \in V_{1} \subset V$. Thus, every point in $K$ has a neighbourhood base of closed and open sets, so $K$ is totally disconnected. 
Next, we prove that (iii) implies (i). Let $f$ be in $X$ with $f(K) \subset[0,1]$. Given $t$ in $K$, we can choose an open and closed neighbourhood $V_{t}$ of $t$ satisfying

$$
\begin{array}{ll}
V_{t} \cap f^{-1}(\{1\})=\emptyset & \text { if } f(t) \neq 1, \\
V_{t} \cap f^{-1}(\{0\})=\emptyset & \text { if } f(t)=1 .
\end{array}
$$

Since $\left\{V_{t}: t \in K\right\}$ is an open (and closed) covering of $K$ there are $t_{1}, \ldots, t_{n}$ in $K$ such that $K=\bigcup_{j=1}^{n} V_{t_{j}}$. The sets

$$
U_{t_{1}}=V_{t_{1}}, \quad U_{t_{2}}=V_{t_{2}} \backslash V_{t_{1}}, \ldots, U_{t_{n}}=V_{t_{n}} \backslash \bigcup_{j=1}^{n-1} V_{t_{j}}
$$

are open and closed, pairwise disjoint, and they are also a covering of $K$.

Define $J=\left\{j \in\{1, \ldots, n\}: U_{t_{j}} \cap f^{-1}(\{0\}) \neq \emptyset\right\}$ and $U=\bigcup_{j \in J} U_{t_{j}}$. Obviously $U$ is open and closed. Now, let us pick arbitrarily a point $t \in K$ and let $j$ in $\{1, \ldots, n\}$ be such that $t \in U_{t_{j}}$. If $f(t)=0$ then $U_{t_{j}} \cap f^{-1}(\{0\}) \neq \emptyset$, and so $j \in J$. Therefore $t \in U$. If $f(t)=1$, then $V_{t_{j}} \cap f^{-1}(\{1\}) \neq \emptyset$ and necessarily $V_{t_{j}} \cap f^{-1}(\{0\})=\emptyset$ (since every $V_{t}$ is disjoint from $f^{-1}(\{1\})$ or $\left.f^{-1}(\{0\})\right)$. Thus $j \notin J$ and consequently $t \in K \backslash U$. This proves that $f^{-1}(\{0\}) \subset U$ and $f^{-1}(\{1\}) \subset K \backslash U$. Since $U$ is open and closed, the function $e: K \rightarrow\{0,1\}$ defined by

$$
e(t)= \begin{cases}0 & \text { if } t \in U \\ 1 & \text { if } t \in K \backslash U\end{cases}
$$

is continuous. In fact, $e \in X$. Furthermore, given $t \in K$,

$$
f(t)=0 \Rightarrow e(t)=0, \quad f(t)=1 \Rightarrow e(t)=1 .
$$

Let $y$ in $X$ satisfy $\varrho(y) \leq 1$ and suppose for the time being that $y(t) \geq 0$ for each $t \in K$. Let us select three real numbers $\lambda, \varepsilon_{1}, \varepsilon_{2}$ such that $0<\lambda<$ $\varepsilon_{1}<\varepsilon_{2}<1 / 2$ and a continuous function $\varphi:[0,1] \rightarrow[0,1]$ satisfying

$$
\varphi(s)= \begin{cases}0 & \text { if } s \leq \varepsilon_{1}, \\ 1 & \text { if } s \geq \varepsilon_{2} .\end{cases}
$$

Then it is clear that the function $f: K \rightarrow \mathbb{R}$ defined by

$$
f(t)= \begin{cases}0 & \text { if } y(t) \leq \varepsilon_{1} \\ \varphi(y(t)) y(t) / \varepsilon_{2} & \text { if } \varepsilon_{1} \leq y(t) \leq \varepsilon_{2}, \\ 1 & \text { if } y(t) \geq \varepsilon_{2}\end{cases}
$$

is continuous. Actually, $f \in X$ and $f(K) \subset[0,1]$. According to the above argument there is a continuous mapping $e: K \rightarrow\{0,1\}$ such that $e(t)=0$ if $f(t)=0$ and $e(t)=1$ if $f(t)=1$. Now, we define $h=(y-\lambda e) /(1-\lambda)$.

If $y(t) \leq \varepsilon_{1}$ then $f(t)=0$ and so $e(t)=0$. It follows that $h(t)=$ $y(t) /(1-\lambda) \in[0,1]$. 
If $\varepsilon_{1} \leq y(t) \leq \varepsilon_{2}$ we have

$$
0 \leq \frac{\varepsilon_{1}-\lambda e(t)}{1-\lambda} \leq \frac{y(t)-\lambda e(t)}{1-\lambda} \leq \frac{y(t)}{1-\lambda} \leq \frac{\varepsilon_{2}}{1-\lambda}<1 .
$$

Therefore, in this case also $h(t) \in[0,1]$.

Finally, if $y(t) \geq \varepsilon_{2}$, then $f(t)=1$ and so $e(t)=1$. We thus have

$$
h(t)=\frac{y(t)-\lambda}{1-\lambda} \in[0,1] .
$$

In this way, it is clear that $h$ belongs to $X$ and $\varrho(h) \leq 1$. Moreover, from the definition of $h$ it follows that

$$
y=\lambda e+(1-\lambda) h .
$$

Note that $e$ is an extreme point of the unit ball of $X$ and that, in case $y(t) \leq 0$, for every $t$ in $K$, the previous reasoning applied to $-y$ provides the same representation of $y$ by means of an extreme point $e$ and a point $h$ of the unit ball of $X$ such that $e(K)=\{0,-1\}$ and $h(K) \subset[-1,0]$.

It remains to treat the case where there are two real numbers $a$ and $b$, with $a<0<b$ and $b-a=1$, such that $a \leq y(t) \leq b$ for every $t$ in $K$.

Under this assumption let us consider the functions $y_{1}, y_{2}: K \rightarrow \mathbb{R}$ given by

$$
y_{1}(t)=\left\{\begin{array}{ll}
y(t) / b & \text { if } y(t) \geq 0, \\
0 & \text { if } y(t) \leq 0,
\end{array} \quad y_{2}(t)= \begin{cases}0 & \text { if } y(t) \geq 0, \\
-y(t) / a & \text { if } y(t) \leq 0,\end{cases}\right.
$$

which are elements of $X$ with $0 \leq y_{1}(t) \leq 1$ and $-1 \leq y_{2}(t) \leq 0$ for every $t$ in $K$. Moreover, it is easy to see that $y=b y_{1}-a y_{2}$. By the above, given $\lambda \in] 0,1 / 2\left[\right.$ there exist $e \in E_{X}$ with $e(K)=\{0,1\}$ and $h \in B_{X}$ with $h(K) \subset[0,1]$ such that $y_{1}=\lambda e+(1-\lambda) h$. Thus

$$
\begin{aligned}
y & =b(\lambda e+(1-\lambda) h)-a y_{2}=b \lambda e+b(1-\lambda) h-a y_{2} \\
& =b \lambda e+(1-b \lambda) \frac{b(1-\lambda) h-a y_{2}}{1-b \lambda} .
\end{aligned}
$$

Set

$$
g=\frac{b(1-\lambda) h-a y_{2}}{1-b \lambda}
$$

and let $t$ be in $K$. If $y(t) \geq 0$,

$$
\frac{a}{1-b \lambda} \leq 0 \leq \frac{b(1-\lambda) h(t)}{1-b \lambda}=g(t) \leq \frac{b(1-\lambda)}{1-b \lambda} .
$$

On the other hand, if $y(t) \leq 0$, we have (using the fact that $y(t) \geq a$ )

$$
\begin{aligned}
\frac{a}{1-b \lambda} & \leq \frac{y(t)}{1-b \lambda} \leq \frac{b(1-\lambda) h(t)+y(t)}{1-b \lambda} \\
& =\frac{b(1-\lambda) h(t)-a y_{2}(t)}{1-b \lambda}=g(t) \leq \frac{b(1-\lambda)}{1-b \lambda} .
\end{aligned}
$$


Therefore, $g(K) \subset\left[\frac{a}{1-b \lambda}, \frac{b(1-\lambda)}{1-b \lambda}\right]$, and hence

$$
\varrho(g) \leq \frac{b(1-\lambda)}{1-b \lambda}-\frac{a}{1-b \lambda}=\frac{1-b \lambda}{1-b \lambda}=1 .
$$

Of course, the previous argument may be performed in an analogous way with the use of a representation of $y_{2}\left(\right.$ instead of $\left.y_{1}\right)$ in the form $\lambda e+(1-\lambda) h$, with $e(K)=\{-1,0\}$ and $h(K) \subset[-1,0]$. Taking into account that $\lambda$ can be chosen in the interval $] 0,1 / 2[$ and $\max \{-a, b\} \geq 1 / 2$, we have proved in fact that for every $\alpha$ in $] 0,1 / 4\left[\right.$ and $y$ in $B_{X}$, there are an extreme point $e$ and an element $g$ in the unit ball of $X$ such that $y=\alpha e+(1-\alpha) g$.

To conclude, let $\alpha$ be in $] 0,1 / 4\left[\right.$ and $x$ in $B_{X}$. According to the above, there are $e_{1} \in E_{X}$ and $g_{1} \in B_{X}$ such that $x=\alpha e_{1}+(1-\alpha) g_{1}$. For the same reason, $g_{1}=\alpha e_{2}+(1-\alpha) g_{2}$ for certain $e_{2} \in E_{X}$ and $g_{2} \in B_{X}$. Proceeding in this manner, we obtain a sequence $\left\{e_{n}\right\}$ of extreme points and a sequence $\left\{g_{n}\right\}$ of elements of the unit ball of $X$ such that $g_{n}=\alpha e_{n+1}+(1-\alpha) g_{n+1}$ for every $n$ in $\mathbb{N}$. Consequently, $x=\alpha e_{1}+(1-\alpha) \alpha e_{2}+(1-\alpha)^{2} \alpha e_{3}+\cdots+(1-\alpha)^{n} \alpha e_{n+1}+(1-\alpha)^{n+1} g_{n+1}$ for each $n \in \mathbb{N}$. From this, it follows immediately that

$$
x=\sum_{n=1}^{\infty}(1-\alpha)^{n-1} \alpha e_{n} .
$$

Since $\sum_{n=1}^{\infty}(1-\alpha)^{n-1} \alpha=1$, it is sufficient to define $\lambda_{n}=(1-\alpha)^{n-1} \alpha$ for every $n \in \mathbb{N}$.

In [1] R. M. Aron and R. H. Lohman introduced the following interesting concepts:

A Banach space $X$ is said to have the $\lambda$-property if, for every $y \in B_{X}$, there are $\lambda \in] 0,1], e \in E_{X}$ and $z \in B_{X}$ such that $y=\lambda e+(1-\lambda) z$. If it is possible to find a common $\lambda$ for all $y$ in $B_{X}$, then $Y$ is said to have the uniform $\lambda$-property.

In view of the preceding proof, it is clear that the space $X$ we have considered has the uniform $\lambda$-property if, and only if, $K$ is totally disconnected. In the last paragraph of the above proof it has been proved that the uniform $\lambda$-property implies the statement (i). This fact is valid for every Banach space (see [1]) and it has been included for the sake of completeness.

To conclude, we note that the previous theorem also holds for spaces of type $C_{0}(L)$, where $L$ is a noncompact, locally compact Hausdorff space. Under these conditions the diameter is a norm (equivalent to the uniform norm) and if $K=L \cup\{\infty\}$ is the one-point compactification of $L$, it suffices to define $t_{0}=\infty$ to observe that $C_{0}(L)$, with the diameter norm, is nothing other than the space $X$ defined in the introduction, for such a selection of $t_{0}$. On the other hand, $L$ is totally disconnected if, and only if, so is its one-point 
compactification. It is perhaps worth remarking that the local compactness of $L$ is important for this last fact. The existence of totally disconnected (not locally compact) spaces whose one-point compactification is connected is well known (see [3]).

Taking into account the above results and comments, we have:

COROLlary 4. Let $L$ be a noncompact locally compact Hausdorff space and $C_{0}(L)$ the space of real continuous functions vanishing at infinity, equipped with the diameter norm. The following statements are equivalent:

(i) For every $x$ in the unit ball of $C_{0}(L)$ there is a sequence $\left\{\lambda_{n}\right\}$ in $[0,1]$ with $\sum_{n=1}^{\infty} \lambda_{n}=1$ and a sequence $\left\{e_{n}\right\}$ of extreme points of $B_{C_{0}(L)}$ such that $x=\sum_{n=1}^{\infty} \lambda_{n} e_{n}$.

(ii) $B_{C_{0}(L)}=\overline{\mathrm{co}}\left(E_{C_{0}(L)}\right)$.

(iii) $L$ is totally disconnected.

In particular, let us observe that $c_{0}$, provided with the diameter norm, has the properties (i) and (ii) of this corollary.

Acknowledgments. This research was supported in part by D.G.E.S., Project MTM 2006-04837 and J.A., Projects FQM 1215 and 1438.

\section{References}

[1] R. M. Aron and R. H. Lohman, A geometric function determined by extreme points of the unit ball of a normed space, Pacific J. Math. 127 (1987), 209-231.

[2] M. Győry and L. Molnár, Diameter preserving linear bijections of $C(X)$, Arch. Math. (Basel) 71 (1998), 301-310.

[3] L. A. Steen and J. A. Seebach, Counterexamples in Topology, Springer, 1978.

Departamento de Álgebra y Análisis Matemático

Facultad de Ciencias Experimentales

Universidad de Almería

04120 Almería, Spain

E-mail: jcnav@ual.es mgsanche@ual.es 